\title{
Heavy Flavored Jets with CMS
}

\author{
Kurt Jung (for the CMS Collaboration) ${ }^{1, \star}$ \\ ${ }^{1}$ University of Illinois at Chicago, 845 W. Taylor St., Chicago, IL 60607
}

\begin{abstract}
The energy loss of jets in heavy-ion collisions is expected to depend on the mass and flavor of the initiating parton. Thus, measurements of jet quenching with tagged partons place powerful constraints on the thermodynamic and transport properties of the hot and dense medium. Furthermore, recent results that constrain the jet production mechanism will shed additional light on the contributions of leading and next-to-leading order heavy flavor jet production with regard to the global energy loss picture. To this end, we present recent results measuring spectra and nuclear modification factors of jets associated to charm and bottom quarks in both $\mathrm{pPb}$ and $\mathrm{PbPb}$ collisions, as well as measurements of dijet asymmetry of pairs of b-jets in $\mathrm{PbPb}$ collisions.
\end{abstract}

\section{Introduction}

The energy loss of highly energetic partons in large collision systems is generally attributed to a process called "jet quenching." Interactions of the jet with the hot and dense medium known as Quark Gluon Plasma, thought to be created in collisions of large ions, induce constituent particle energy loss and jet broadening, where constituent particles are pushed far from the jet axis. Both features contribute significantly to the measured jet energy modification. Measurements of jet quenching are expected to depend on parton flavor primarily through a modification of two jet quenching mechanisms: radiative and collisional energy loss $[1,2]$, as both these processes are thought to include mass-dependent effects $[3,4]$. A process known as the dead cone effect suppresses forward gluon radiation in an angle proportional to the mass of the parton, and collisional energy transfer to the medium is mitigated somewhat by the heavy parton mass. In addition to these effects, a typical inclusive-jet measurement contains a large fraction of jets seeded by high- $p_{\mathrm{T}}$ gluons, while a measurement of tagged b-jets, for example, will contain significantly fewer gluon-seeded jets. Under the assumption that radiative energy loss is the dominant component of jet quenching, gluon jets are expected to quench more strongly than quark jets, due to the larger color factor for gluon emission from gluons than from quarks [5].

The heavy flavored jet measurements summarized in these conference proceedings include finalized measurements of charm-tagged jets [6] in proton-lead $(\mathrm{pPb})$ collisions, as well as measurements of di-b-jets [7] in lead-lead ( $\mathrm{PbPb})$ collisions. Measurements of jet quenching in $\mathrm{pPb}$ collisions are not expected to include effects from the QGP and are made primarily to test effects of initial-state "cold nuclear matter" processes on jet production. The presented inclusive charm quark jet cross-section measurement is the first of its kind and will shed light on the nature of the charm jet production and

\footnotetext{
^e-mail: kurtejung@gmail.com
} 
alllow further exploration of the charm-tagged sector at high energies. Measurements of di-b-jets in $\mathrm{PbPb}$ attempt to clarify the magnitude of heavy flavored jet energy loss at leading-order, as opposed to inclusive b-jet studies, which are dominated by next-to-leading order production graphs [8]. These next-to-leading order processes like gluon splitting, where a highly energetic gluon splits into a b $\bar{b}$ pair, are expected to contribute significantly to an inclusive b-jet measurement. While interesting in their own right, the back-to-back selection of b-jets will preferentially select leading-order b-jet production to study $\mathrm{b}$ quarks that interact directly with the medium.

\section{Identification of Flavored Jets}

Jets containing heavy quarks are tagged via combinations of quantities that relate to the relatively long lifetimes of B and D mesons. For jet spectra measurements, reconstructed secondary vertices are used to identify both b- and c-jets. The b-jets are tagged through associating the jet to a secondary vertex that has at least two associated tracks and a flight distance significance greater than 2 standard deviations away from the primary vertex. The c-jets are tagged similarly, associating the jets to a secondary vertices with a looser flight distance significance of least 1.68 standard deviations away from the primary vertex, though these vertices are required to have at least three tracks. Once the jets are tagged, the tagging purity is calculated using template fits of both tagged and untagged jets to distributions of the secondary vertex mass. The template fits for the c-jet analysis use a modification of the secondary vertex mass that includes missing energy from neutral particles and neutrinos. If the secondary vertex displacement vector is not parallel to the momentum vector of the vertex, an additional energy is added to the vertex mass to account for the undetected momentum. The correction for missing particles allows enough discrimination power between b-and c-jets such in the template fits so that all three flavor contributions (light, c, and b) are accurately calculated simultaneously in a sample. The maximum b-tagging purity achieved is roughly $60 \%$, while the maximum c-tagging purity is roughly $30 \%$. For further details, see the b-jet [9] and c-jet [6] publications.

The b-dijet analysis uses a more advanced b-jet identification strategy, using machine learning algorithms that effectively combine information from secondary vertices, leptons, and charged particles. A boosted decision tree is used to optimize combinations of more than 29 different parameters such that the b-jet sample purity is upwards of $90 \%$. An additional light jet rejection is imposed by calculating the yields of dijet pairs close together in azimuthal angle $(\Delta \phi<\pi / 3)$ and removing this yield across the entire $\Delta \phi$ range. This subtraction exploits the fact that while the b-jet $\Delta \phi$ distributions peak around $\Delta \phi=\pi$, the light jet pairs are generally combinatorial in nature and have a flat $\Delta \phi$ distribution. Further corrections are applied to account for situations where light jets are mistagged as the away-side partner of a leading b-jet. These inefficiencies are highly $p_{\mathrm{T}}$ and centrality dependent, ranging from about $50 \%$ at $40 \mathrm{GeV}$ to very small values $(<1 \%)$ at $100 \mathrm{GeV}$. This correction is derived by inverting the b-tagging selection and subtracting the inverted yields from the signal, where the inverted yield is weighted by the mistagging fraction, as derived in Monte Carlo simulations. More details can be found in the preliminary document [7].

\section{Results}

The subleading-to-leading-jet $p_{\mathrm{T}}$ ratio $\left(x_{j}\right)$ ranges from 0 to 1 and depends on the jet kinematic selection. The di-b-jet measurement selects leading jets of at least $100 \mathrm{GeV}$ and subleading jets of at least $40 \mathrm{GeV}$, separated in azimuthal angle by more than $\Delta \phi>2 \pi / 3$. This kinematic selection leads to an average $\left\langle x_{j}\right\rangle$ of around 0.7 for $\mathrm{b}$-jets in pp collisions and between 0.6 and 0.7 for $\mathrm{b}$-jets in $\mathrm{PbPb}$ collisions, depending on collision centrality. These $\left\langle x_{j}\right\rangle$ values can be compared to inclusive 
jet measurements, where similar values are found for all collision centralities, indicating no significant quenching differences between leading-order inclusive dijets and di-b-jets, shown in Fig. 1 (left). We observe a significant centrality dependent trend for $\left\langle x_{j}\right\rangle$, indicating the presence of jet quenching, however the magnitude and shape of this trend is very similar for both the dijet samples studied. This is directly compared in Fig. 1 (right), which shows the ratios of the $x_{j}$ values for b-jets to inclusive jets. The largest separation is in the 10-30\% centrality bin, which shows a separation of about 1.3 standard deviations. The similarity of the inclusive dijet and di-b-jet measurements strengthen the conclusions that high- $p_{\mathrm{T}}$ b-jets behave very similarly to high- $p_{\mathrm{T}}$ inclusive jets, first observed by single b-jet measurements at CMS $[9,10]$.
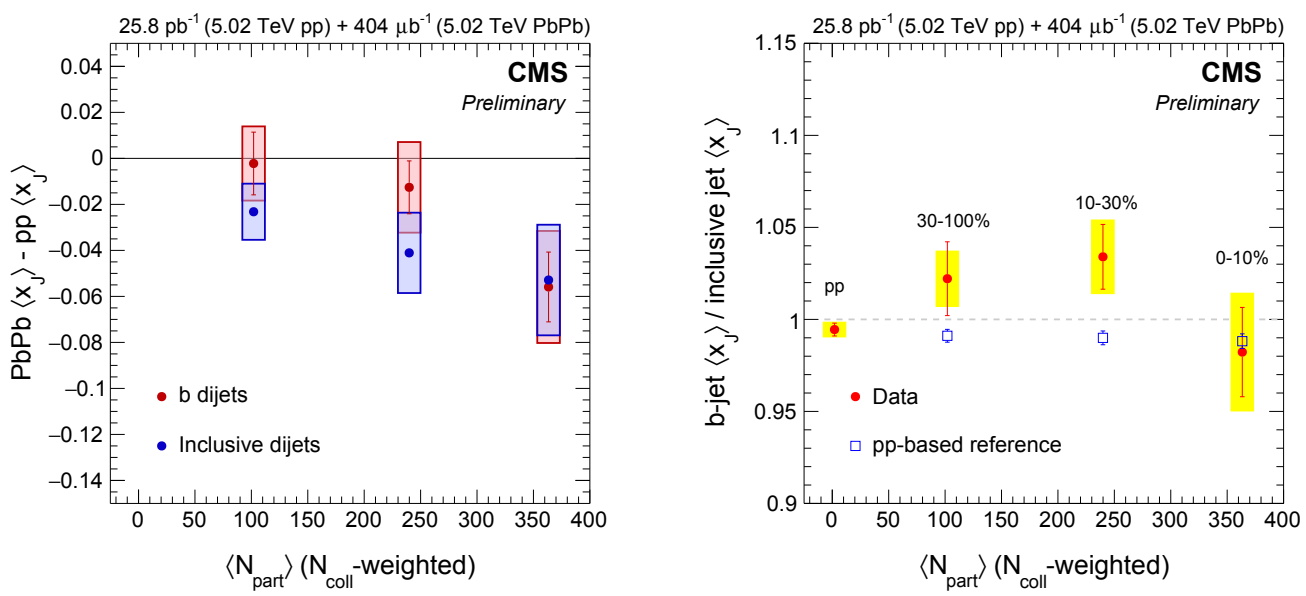

Figure 1. The left figure shows the difference of the average $x_{j}$ values of dijets between $\mathrm{PbPb}$ and pp collisions for inclusive-jets (blue) and for b-jets (red). The right figure shows the ratio of the average $x_{j}$ values between $\mathrm{b}$-jets and inclusive-jets. Both figures show distributions as a function of the average number of collision participants, weighted by the average number of binary collisions [7].

Smaller system measurements are also presented in these proceedings. Measurements of jets in collision systems like $\mathrm{pPb}$ are expected to probe nuclear effects in the initial-state (pre-collision), as a QGP medium is not expected to be produced. To study these initial-state effects, a quantity known as $R_{\mathrm{pA}}$ is measured, comparing the yields of jets in $\mathrm{pPb}$ collisions to pp collisions. The $R_{\mathrm{pA}}$ value is defined as:

$$
R_{\mathrm{pA}}=\frac{1}{A} \frac{d \sigma_{P b P b} / d p_{\mathrm{T}}}{d \sigma_{p p} / d p_{\mathrm{T}}},
$$

where $\mathrm{A}$ is the nuclear mass number of $\mathrm{Pb}(208)$, and is included in order to account for the geometrical enhancement of jet production based on the additional number of hard scatterings in $\mathrm{pPb}$ collisions relative to $\mathrm{pp}$ collisions. The $R_{\mathrm{pA}}$ is measured for both b-jets and c-jets in Fig. 2 on the left and lower right panels, respectively. Both measurements show consistency with unity, which indicates that the nuclear initial-state has only a minor effect on jet production at high- $p_{\mathrm{T}}$. Note especially that the b-jet measurement is convoluted somewhat by the use of a PутніA 6 simulation [11] instead of true pp data, due to the fact that $5 \mathrm{TeV}$ pp data was not yet available when the measurement 
was published. To account for this, a $22 \%$ global uncertainty was assigned to the measurement to account for any differences between the pp data and the PYтніA simulation. This factor was derived by interpolating the adherence of the PутніA calculation to data at both 2.76 and $7 \mathrm{TeV}$. When these uncertainties are taken into account, both the b-jet and c-jet $R_{\mathrm{pA}}$ values are consistent with both unity and each other. Finally, these measurements can be compared to the inclusive-jet $R_{\mathrm{pA}}$, as measured by the CMS Collaboration [12]. The $R_{\mathrm{pA}}$ of all three jet flavors are consistent with one another across the entire kinematic range, indicating the lack of sensitivity required to probe the flavor dependence of initial-state jet energy modification.
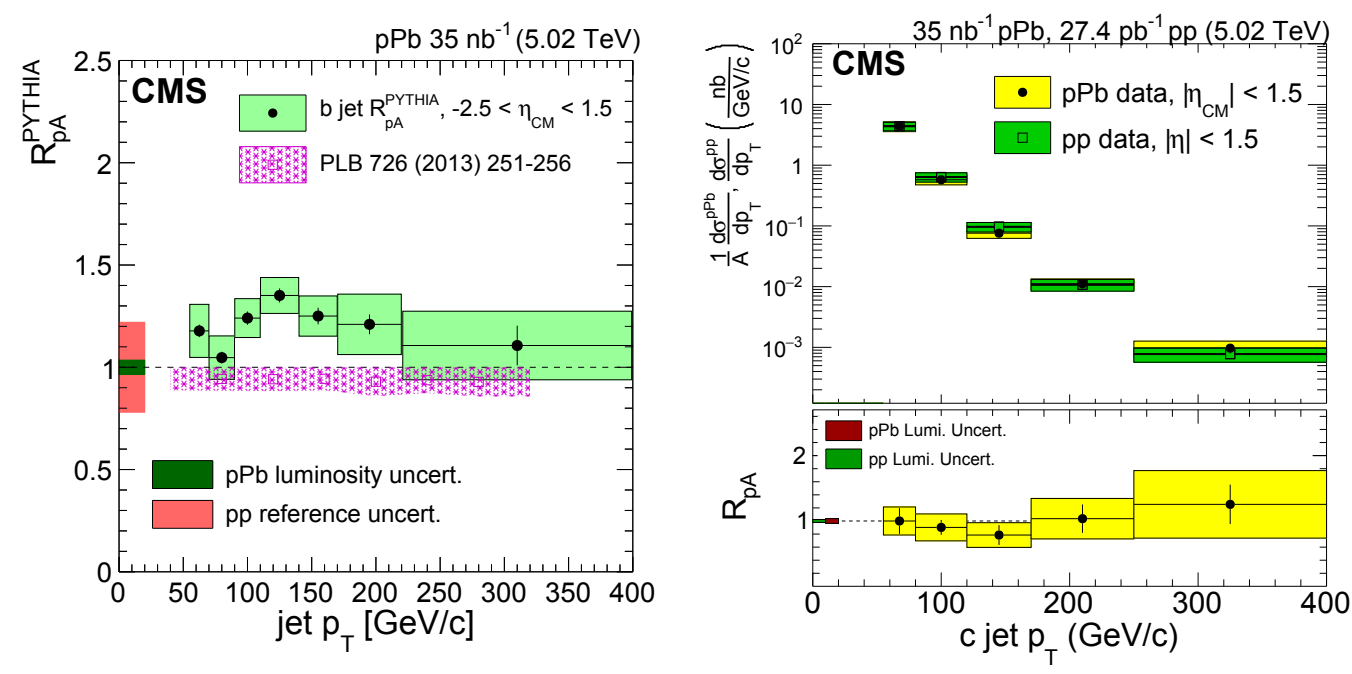

Figure 2. The $R_{\mathrm{pA}}$ values for b-jets (left [9]) and c-jets (right, lower panel [6]) are shown as a function of jet $p_{\mathrm{T}}$. The b-jet measurements are compared to a prediction including only energy-loss effects from Vitev., et. al. [3]. The upper panel of the right figure shows the $\mathrm{c}$-jet cross-sections in $\mathrm{pp}$ and $\mathrm{pPb}$, where the $\mathrm{pPb}$ spectrum is scaled by the mass number of $\mathrm{Pb}$. Also shown are uncertainties from luminosity on both figures, as well as a pp reference uncertainty on the b-jet measurement.

\section{References}

[1] H. van Hees, R. Rapp, Phys. Rev. C 71, 034907 (2005), nucl-th/0412015

[2] G.D. Moore, D. Teaney, Phys. Rev. C 71, 064904 (2005), hep-ph/\$412346

[3] J. Huang, Z.B. Kang, I. Vitev, Phys. Lett. B 726, 251 (2013), 1306.0909

[4] D.K. Yu.L. Dokshitzer, Phys. Lett. B 519, 199 (2001), hep-ph/0106202

[5] D. d'Enterria, Jet quenching (Springer Materials, 2010), Vol. 23, chap. 6.4, 0902.2011

[6] V. Khachatryan et al. (CMS), Phys. Lett. B 772, 306 (2017), 1612 . 08972

[7] CMS Collaboration (CMS) (2016), http://cds.cern.ch/record/2202805

[8] A. Banfi, G.P. Salam, G. Zanderighi, JHEP 07, 026 (2007), 0704 . 2999

[9] V. Khachatryan et al. (CMS), Phys. Lett. B 754, 59 (2016), 1510.03373

[10] S. Chatrchyan et al. (CMS), Phys. Rev. Lett. 113, 132301 (2014)

[11] R. Field, Acta Phys. Polon. B 42, 2631 (2011), 1110. 5530

[12] V. Khachatryan et al. (CMS), Eur. Phys. J. C 76, 372 (2016), 1601.02001 